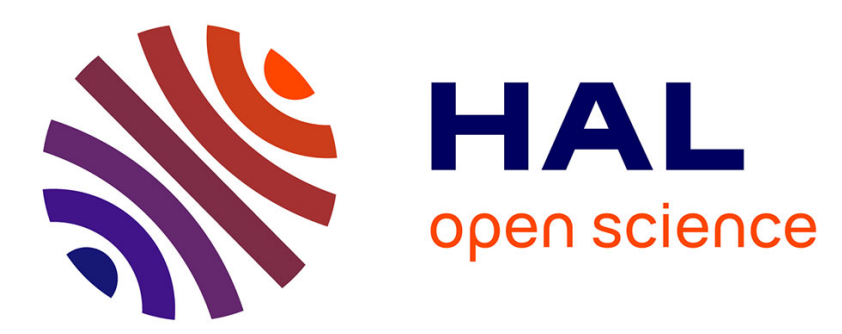

\title{
Osteological connections of the petrosal bone of the extant Hippopotamidae Hippopotamus amphibius and Choeropsis liberiensis
}

\author{
Maeva Orliac, Franck Guy, Renaud Lebrun
}

\section{- To cite this version:}

Maeva Orliac, Franck Guy, Renaud Lebrun. Osteological connections of the petrosal bone of the extant Hippopotamidae Hippopotamus amphibius and Choeropsis liberiensis. MorphoMuseum, 2014, 1 (1), pp.e1. 10.18563/m3.1.1.e1 . hal-01902601

\section{HAL Id: hal-01902601 \\ https://hal.science/hal-01902601}

Submitted on 26 Oct 2018

HAL is a multi-disciplinary open access archive for the deposit and dissemination of scientific research documents, whether they are published or not. The documents may come from teaching and research institutions in France or abroad, or from public or private research centers.
L'archive ouverte pluridisciplinaire HAL, est destinée au dépôt et à la diffusion de documents scientifiques de niveau recherche, publiés ou non, émanant des établissements d'enseignement et de recherche français ou étrangers, des laboratoires publics ou privés. 


\title{
Osteological connections of the petrosal bone of the extant Hippopotamidae Hippopotamus amphibius and Choeropsis liberiensis
}

\author{
ORLIAC M.J*, GUY F. $\dagger$, LEBRUN R.* \\ * Laboratoire de Paléontologie, Institut des Sciences de l'Évolution de Montpellier (ISE-M, UMR 5554, CNRS, UM2, IRD), c.c. \\ 064, Université Montpellier 2, place Eugène Bataillon, F-34095 Montpellier Cedex 05, France \\ $\dagger$ Université de Poitiers - UFR SFA, iPHEP UMR CNRS 7262, Bât B35 - TSA 51106, 6 rue Michel brunet, 86073, Poitiers Cedex \\ 9, France
}

\begin{abstract}
This project presents the osteological connections of the petrosal bone of the extant Hippopotamidae Hippopotamus amphibius and Choeropsis liberiensis by a virtual osteological dissection of the ear region. The petrosal, the bulla, the sinuses and the major morphological features surrounding the petrosal bone are labelled, both in situ and in exploded models presenting disassembly views. The directional underwater hearing mode of Hippopotamidae is discussed based on the new observations.
\end{abstract}

Key words: ear region, artiodactyla, bulla, sinus, hippos

Submitted 25.09.2014, Accepted 16.10.2014

(C) Copyright Maeva Orliac October 2014

TECHNICAL AND SPECIMEN-RELATED PARAMETRES

\begin{tabular}{|l|l|l|}
\hline Specimen number & UPPal-M09-5-005a & UM N179 \\
\hline Species & Choeropsis liberiensis & Hippopotamus amphibius \\
\hline Repository institution & Université de Poitiers, France & Université de Montpellier 2, France \\
\hline CT scanner institution & Université de Poitiers, France & Université de Poitiers, France \\
\hline$(\mathrm{CT})$ scanner model & Viscom X8050-16 & Rx Solutions EasyTom150 \\
\hline$(\mathrm{CT})$ scanner operator & F. Guy & F. Guy \\
\hline Voxel size & $0.109 x 0.109 x 0.109$ & $0.151 x 0.151 x 0.151$ \\
\hline Number of slices & 909 & 1291 \\
\hline Slices dimensions & $943 x 943$ & $1291 x 1162$ \\
\hline Author of 3D model & M.J. Orliac & M.J. Orliac \\
\hline Model ID number & $\begin{array}{l}\text { M3\#1_UPPal-M09-5-005a } \\
\text { M3\#2_UPPal-M09-5-005a }\end{array}$ & $\begin{array}{l}\text { M3\#3_UM N179 } \\
\text { M3\#4_UM N179 }\end{array}$ \\
\hline
\end{tabular}

\section{METHODS}

AVIZO 6.3 (Visualization Sciences Group) software was used for visualization, segmentation and $3 \mathrm{D}$ rendering. The different structures were extracted using the segmentation tools brush with the same limited range values, and were segmented manually in separate labelfield modules. The 3D models are provided in .vtk format. They were combined in two different projects of ISE-MeshTools (Lebrun, 2014) software. Projects were also labelled and coloured with this software. Labels are provided in .flg format.

Nomenclature of morphological structures follows mainly follows O’Leary 2010 and Wible 2011, whereas nomenclature of sinuses follows the recent work of Hoffmann et al. 2014 on the ovine cerebral venous system.

\section{INTRODUCTION}

Extant members of the species Hippopotamus amphibius vocalize underwater a water-born sound and are able to respond to the directionality of sounds underwater (Barklow, 1994, 1997, 2004) which implies a specialization of the sound perception pathway. Based on what is known for cetaceans, reduction of the mastoid region of the petrosal bone and inflation of the tegmen tympani have been hypothesized to play a role in directional underwater hearing in hippos (Luo 
\& Gingerich, 1999; O’Leary et al., 2012). The petrosal bone of extant hippos has been described in detail by O'Leary 2010 , but their auditory region has not been investigated in its entirety, and the precise bony connections of the petrosal bone in the cranium remains, to our knowledge, undescribed in the literature.

Here is reconstructed in situ the petrosal of Hippopotamus amphibius and Choeropsis liberiensis based on microCT scanning of the specimen UM N179 curated at the Universite des Sciences de Montpellier, France and UPPal-M09-5-005a curated at the Université de Poitiers, France, respectively. In the associated 3D models, the petrosal, the bulla, the sinuses and the major morphological features surrounding the petrosal bone are labelled, both in situ (Choeropsis liberiensis, M3\#1 UPPal-M09-5-005a; Hippopotamus amphibius, M3\#3 UM N179) and in exploded models presenting disassembly views Choeropsis liberiensis, M3\#2_UPPal-M09-5-005a; Hippopotamus amphibius, M3\#4_UM N179).

The present aim is to describe the connections of the petrosal with the other bones of the cranium and to propose a hypothesis regarding the functional role of the inflated tegmen tympani in hippos. Besides, this very area is a potential source of important phylogenetic characters (e.g. Geisler \& Luo, 1998; Geisler \& Huen, 2003; Geisler et al., 2007; O'Leary \& Gatesy, 2008) and detailed description of the auditory region of extant hippopotamidae might be of interest for future phylogenetic studies.

\section{DESCRIPTION AND DISCUSSION}

\section{Location of the petrosal and contacts with other features of the braincase}

The petrosal is large relative to the size of the braincase. It is situated between the cerebral and cerebellar part of the braincase. In medial (Fig 1A-B) and anterior (Fig 1C-D) views, the lateral surface of the tegmen tympani constitutes the floor of the temporal sinus (see below), whereas its medial surface is most probably in contact with the cerebellum. The edge between the lateral and medial surfaces of the tegmen tympani (crista petrosa, Wible et al., 1995) prolongs the slight inflection of bone of the internal wall of the braincase corresponding to the crista tentorica. This location of the petrosal greatly differs from that of suoids where the petrosal completely lies posterior to the crista tentorica.

As already described in the literature (O'Leary, 2010), the mastoid region of the petrosal of adult specimens of Hippopotamus amphibius and Choeropsis liberiensis has no occipital exposure (amastoidy) and does not intercalate between the squamosal and the occipital bones. As a result, contacts of the petrosal bone with other bones of the braincase are reduced. In both species, the petrosal contacts the squamosal on its lateral side, at the level of the tegmen tympani (Figs 2, $3)$. A large sinus separates the petrosal from the squamosal on a wide surface. This sinus is dorsally enclosed by a thin flake of the parietal bone projecting ventrally on the internal surface of the braincase (Fig 1A-B, Fig 2A-C). Based on the ovine cerebral venous system (Hoffmann et al., 2014), this sinus would mainly correspond to the temporal sinus cavity (pathway for the capsuloparietal emissary vein, Wible, 2011). Part of it opens dorsally well above the zygomatic process, close to the nuchal crest (temporal foramina = foramina for rami temporales, figure 1C; see Giannini et al., 2006). Posteriorly, the temporal sinus cavity connects to the sigmoid sinus pathway (corresponding to the posterior distributary branch of the transverse sinus; Wible, 1993); anteriorly, it opens on the ventral surface of the cranium, medial to the glenoid surface. This anterior canal corresponds to the pathway of the capsuloparietal emissary vein which should exit the cranium anteriorly between the squamosal and the petrosal, through a space that would correspond to the location of the postglenoid foramen (ventral aperture of the capsuloparietal emissary vein, Wible, 1990, Geisler \& Luo, 1998). Neither in Hippopotamus, nor in Choeropsis does it correspond to a real (fully individualized) foramen (Fig $1 \mathrm{G}-\mathrm{H})$, as mentioned by Geisler et al. (2007). Medial to the temporal sinus cavity lies a pathway for possible tributary branches of the capsuloparietal emissary vein system. In the specimens investigated here, these tributary branches canals open ventrally via two small openings situated posterior to the postglenoid process (Fig. 1G-H). These two canals gather in a larger one that opens dorsally, either on the lateral surface of the zygomatic process in Hippopotamus amphibius (suprameatal foramen Fig 1C-D) or just anterior to the zygomatic process, at the root of the zygomatic arch in Choeropsis liberiensis (Fig. 1E-F). Ventrally, the anterior-most foramen corresponds to the opening of the main pathway of the capsuloparietal emissary vein and should be considered as the postglenoid foramen per se (Fig. 1G-H), though not completely individualized. The term "secondary postglenoid foramen" has been proposed for an opening located lateral to the glenoid fossa on the petrosal/squamosal suture (Geisler et al., 2007) and described in fossil hippopotamoids (Geisler et al., 2007, Orliac et al., 2013), while an opening located posterior to the postglenoid process has been recognised as the postglenoid foramen by Orliac et al. (2013). If the same pattern of sinus occurs in fossil hippopotamoids, then the foramen medial to the glenoid fossa would correspond to the postglenoid foramen, and the foramina posterior to the postglenoid process would be openings of the tributary branches of the capsuloparietal emissary vein system.

The wide temporal and sigmoid sinuses isolate a large part of the petrosal from the other bones of the braincase. The petrosal bone however shows tight contact/fusion with elements of the auditory region. The tegmen tympani intercalates and is pinched between the external meatal tube of the tympanic bulla ventromedially and the squamosal at the root of the craniomandibular joint dorsolaterally. The tegmen tympani tightly contacts the tympanic bulla, at the level of the lateral wall and of the external meatal tube (Fig. 2-3). In both species, the mastoid region and the ventrolateral tuberosity of the petrosal enclose the dorsomedial border of the external meatal tube. The latter is strongly attached to the squamosal by a bony infilling enclosing the space between the posterior edge of the glenoid surface and the postympanic process of the squamosal (Fig 2-3). This structure, also found in suoids, is recognized as the "tympanic neck" by Pearson (1927). The latter prolongs the external meatal tube by a long bony structure joining the 


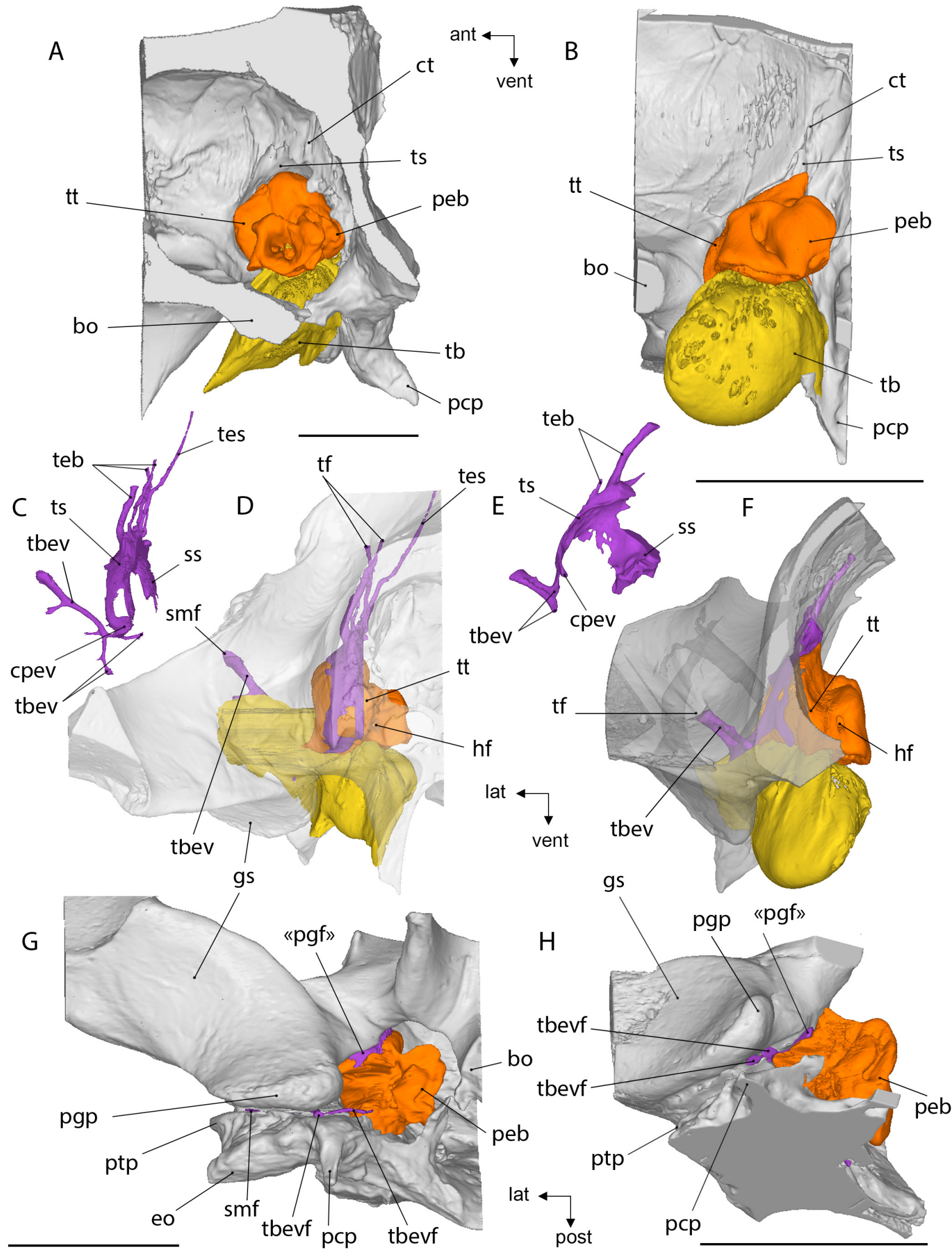

Fig. 1 - Location of the right petrosal bone in the braincase of Hippopotamus amphibius (A, C-D, G; M3\#3_UM N179) and Choeropsis liberiensis (B, E-F, H; M3\#1_UPPal-M09-5-005a) illustrated in A-B medial, C-F anterior, G-H ventral views with bulla removed, when reconstructed, sinuses are coloured in purple. Abbreviations: bo, basioccipital; cpev, canal of capsuloparietal emissary vein; ct, crista tentorica; eo, exo occipital; gs, glenoid surface; hf, hiatus fallopii; iam, internal acoustic meatus; pcp, paracondylar process; peb, petrosal bone; pgf, postglenoid foramen; pgp, postglenoid process; ptp, postympanic process; smf, suprameatal foramen; ss, sigmoid sinus; tb, tympanic bulla; tbev, canal of tributary branch of capsuloparietal emissary vein; tbevf, tributary branch of capsuloparietal emissary vein foramen; teb, temporal branche; tes, transverse sinus; tf, temporal foramen; ts, temporal sinus; tt, tegmen tympani. Scale bars $=5 \mathrm{~cm}$. 


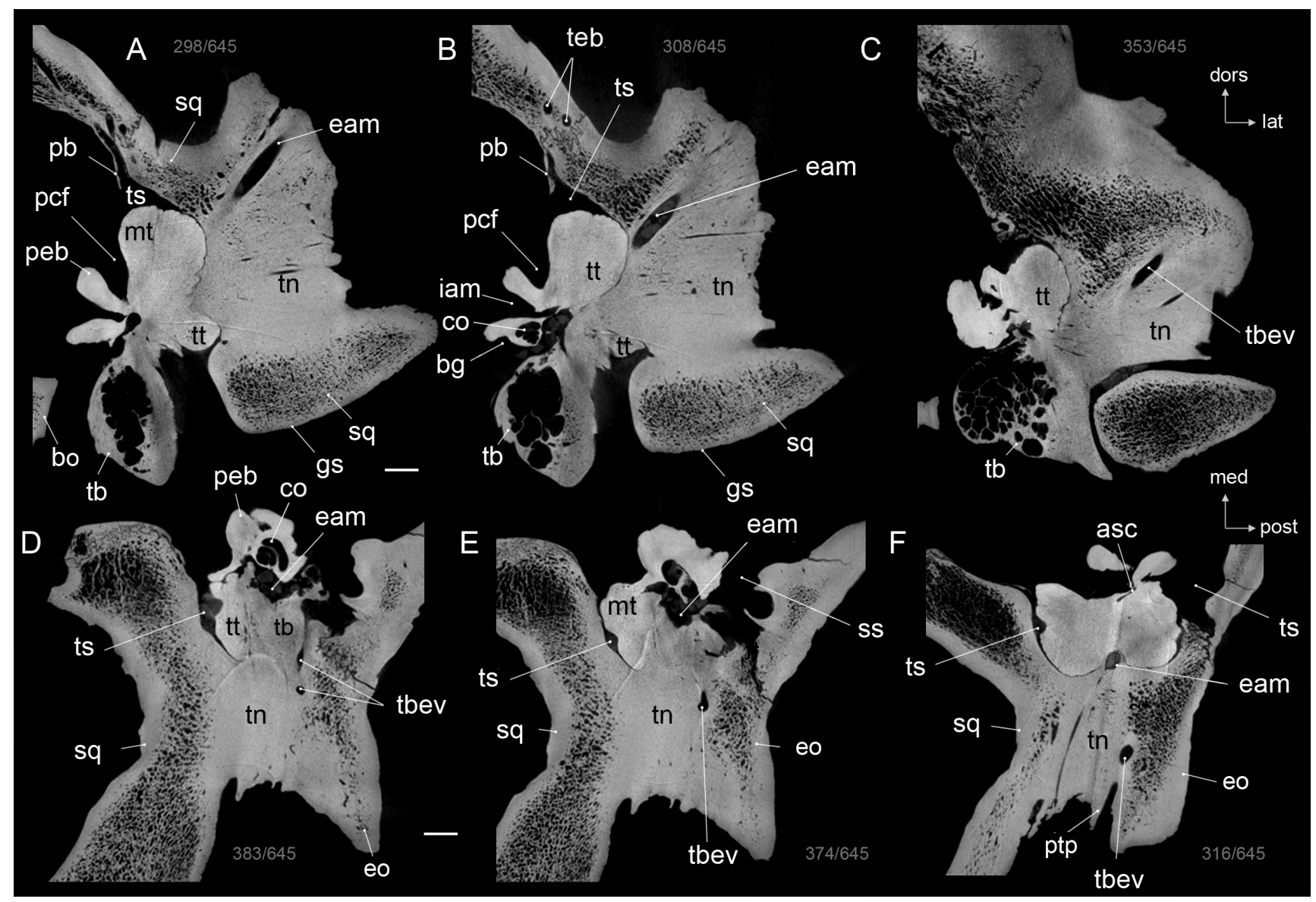

Fig. 2 - CT image through the ear region of the skull of Hippopotamus amphibius (UM N179), showing the connexions and internal anatomy of the right petrosal. A-C coronal sections; D-F, transverse sections. Abbreviations: asc, anterior semicircular canal; bg, basicapsular groove; bo, basioccipital; co, cochlear canal; eam, external auditory meatus; eo, exo occipital; gs, glenoid surface; mt, mastoid; pb, parietal bone; pcf, prefacial commissure fossa; pcp, paracondylar process; peb, petrosal bone; ptp, postympanic process; sq, squamosal; ss, sigmoid sinus; tb, tympanic bulla; tbev, canal for tributary branch of capsuloparietal emissary vein; teb, temporal branche; tn, tympanic neck; ts, temporal sinus; tt, tegmen tympani. Scale bars $=1 \mathrm{~cm}$.

postympanic process. This "false external meatal tube" places the external auditory meatus in a dorsal position.

\section{Directional underwater hearing in hippopotamids}

The skull of living hippos, especially that of Hippopotamus, show a peculiar disposition of the different orifices, with the eyes, ears, and nostrils in a high position. While the rest of their body is below water, they are able to breathe and see above the water surface, but also to communicate via "amphibious communication" (Barklow, 2004). According to Barklow's recordings, Hippopotamus can communicate via grunts, underwater screams, underwater clicks, croaks and whines given without the expiration of air (Barklow, 1997), as well as nasal flutters and plosive blows (produced by nasal valves). Hippopotamus produces sounds audible underwater only, simultaneous amphibious sounds, and sounds audible in air only. Barklow (2004) noticed that with their head in the amphibious position, hippos could respond to sounds emitted underwater that were inaudible in air, suggesting that with their ear outside the water surface, they are able to hear in air and water simultaneously.
H. amphibius also responds to the directionality of underwater sounds (Barklow, 2004). However, for terrestrial mammals, the direction of a sound is determined by the differences of acoustic stimuli received by the two independent ears, and by the interaural time difference (Reysenbach de Haan, 1960; Nummela \& Thewissen, 2008). In water, sound travels five times faster than in air, reducing both interaural time and intensity differences. Besides, intensity difference of the stimuli received by the two ears underwater is reduced because of omnidirectional conduction of sounds through the tissues of the head to both ears. The ability of $H$. amphibius to directionally hear sound underwater therefore implies a specialization of the sound perception pathway. Underwater directional hearing implies a drastic limitation of the transmission of skull vibrations to the ear region. In turn, incoming sound waves must be confined to specialized, directionally specific channels (Ketten, 1994).

Much fewer information is available for Choeropsis liberiensis regarding its ability to hear sound directionally underwater. Hippotamus calls are associated to gregarious behaviour, and one can wonder if directionally underwater hearing exists in a solitary species like Choeropsis, although 


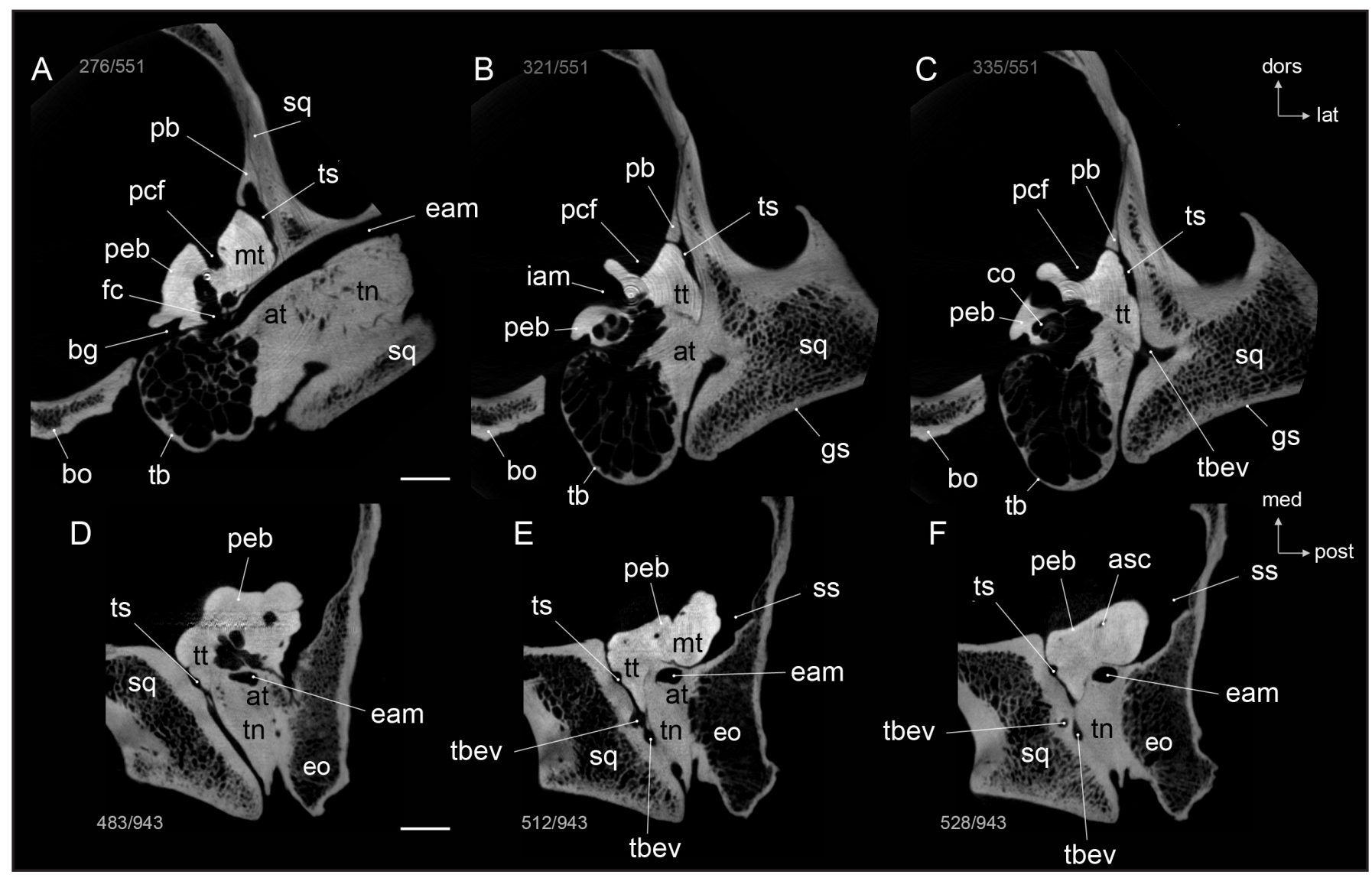

Fig. 3 - CT image through the ear region of the skull of Choeropsis liberiensis (UPPal-M09-5-005a), showing the connexions and internal anatomy of the right petrosal. A-C coronal sections; D-F, transverse sections. Abbreviations: asc, anterior semicircular canal; at, auditory tube of the tympanic bulla; bg, basicapsular groove; bo, basioccipital; co, cochlear canal; eam, external auditory meatus; eo, exo occipital; gs, glenoid surface; iam, internal acoustic meatus; mt, mastoid; pb, parietal bone; pcf, prefacial commissure fossa; pcp, paracondylar process; peb, petrosal bone; ptp, postympanic process; sq, squamosal; ss, sigmoid sinus; tb, tympanic bulla; tbev, canal for tributary branch of capsuloparietal emissary vein; teb, temporal branche; tn, tympanic neck; ts, temporal sinus; tt, tegmen tympani. Scale bars $=1 \mathrm{~cm}$.

phylogenetically closely related to Hippopotamus amphibius (estimated divergence age $=5.7$ Ma, Montgelard et al., 1997 ).

Modern cetaceans reduce the bone conduction by an isolation of the tympanoperiotic complex by means of air sinuses (Nummela et al., 2007). In modern hippos, the fact that the small mastoid region of the petrosal does not intercalate between the squamosal and the occipital bone greatly reduces the contact between the petrosal and the braincase, as the petrosal is no longer part of the braincase per se. Then, as described by O'Leary et al. (2012) based on CT scan slides, the petrosals of the specimens of Hippopotamus amphibius and Choeropsis liberiensis are isolated from the lateral wall of the braincase by large temporal and sigmoid sinuses which also contribute to eliminate omnidirectional conduction of sounds. On the other hand, in both extant species the petrosal has close relationships with the tympanic bulla and tympanic neck (Figs. 2, 3). In Choeropsis the ventrolateral tuberosity of the tegmen tympani intercalates and is pinched between the lateral wall of the auditory bulla (or the external auditory meatus tube) ventromedially, and the squamosal at the root of the cranio-mandibular joint dorsolaterally. In the specimen of $H$. amphibius examined here, the tegmen tympani has a very tight and wide contact with the bulla and the tympanic neck (Fig. 2B). Therefore these observations show that, if the petrosal is dorsomedially isolated by large sinuses, it entertains very tight connections with the area surrounding the glenoid surface.

Luo and Gingerich (1999:p. vii) proposed that "substantial capacity for directional hearing in water" in cetaceans resulted from the pachyostotic and osteosclerotic characteristics of their petrosal. O'Leary et al. (2012) showed that hippos and cetaceamorphans indeed had pachyostotic tegmen tympani compared to fully terrestrial artiodactyls. The CT scan images of the specimens examined here not only show that the tegmen tympani is pachyostotic, but also that it forms, together with the lateral wall of the bulla, the external meatal tube and the tympanic neck, a "pachyostotic complex" located at the root of the cranio-mandibular joint. Besides, the bone of the petrosal /lateral wall of the bulla, and meatal tube/tympanic neck complex is very compact, much more than that of the surrounding features (Figs. 2-3). Barklow (2004) proposed that hippos used bone conduction for underwater sound perception using their large lower jaw as "a path for resonant conduction of underwater sound to the squamosal bone and then to the tympanic and periotic bones" (Barklow, 2004: 1131). Based 
on the present osteotogical observations, there is no clear clue to precise how bilateral conduction might be realized in hippos. However, the specialization of the compact tegmen tympani/bulla/tympanic neck complex show that it is most probably involved in underwater directional hearing in hippos, either by creating a barrier for sound penetration and helping in isolating the ear acoustically, or by providing a pathway for the sound to the middle ear.

\section{ACKNOWLEDGMENTS}

We thank S. Jiquel (Université Montpellier2, ISEM) for access to the collections and specimen preparation. Many thanks to G. Billet for discussion on ear region morphology. We are greatful to the Microtomography facility of the université de Poitiers. This is ISEM publication 2014-182.

\section{BIBLIOGRAPHY}

Barklow, W. E., 1994. Big talkers. Wildlife Conservation 97, 2029.

Barklow, W. E., 1997 Some underwater sounds of the hippopotamus (hippopotamus amphibius). Marine and Freshwater Behaviour and Physiology 29(1-4), 237-249.

Barklow, W. E., 2004. Amphibious communication with sound in hippos, Hippopotamus amphibius. Animal behaviour $68,1125-1132$

Geisler, J. H., and Z. Luo, 1998. Relationships of Cetacea to terrestrial ungulates and the evolution of cranial vasculature in Cete. In Thewissen, J.G.M. (Ed.), The emergence of whales. New York: Plenum Press, pp. 163-212.

Geisler, J. H., Huen, M. D., 2003. Morphological Support for a Close Relationship between Hippos and Whales. Journal of Vertebrate Paleontology 23(4), 991-996.

Geisler, J. H., Theodor, J. M., Uhen, M. D., Foss, S. E., 2007. Phylogenetic relationships of cetaceans to terrestrial artiodactyls. In Prothero, D. R, Foss, S. E (Eds.), The Evolution of Artiodactyls. Baltimore: The Johns Hopkins University Press, pp. 19-31.

Giannini N, P., Wible, J. R., Simmons, N. B., 2006. On the cranial osteology of Chiroptera. I. Pteropus (Megachiroptera: Pteropodidae). Bulletin of the American Museum of Natural History 295, 134 pp.

Hoffmann, A., Stoffel, M. H., Nitzsche B., Lobsien D., Seeger J., et al., 2014. The Ovine Cerebral Venous System: Comparative Anatomy, Visualization, and Implications for Translational Research. PLoS ONE 9(4), e92990. doi:10.1371/journal.pone.0092990.

Irwin, D. M., Kocher, T. D., Wilson, A. C., 1991. Evolution of the cytochrome $b$ gene of mammals. Journal of Mammalian Evolution 32, 128-144.

Ketten, D. R., 1994. Functional analysis of whale ears: Adaptations for underwater hearing. I.E.E.E. Proceedings in Underwater Acoustics 1, 264-270.

Lebrun, R., 2014. ISE-MeshTools, a 3D interactive fossil reconstruction freeware. 12th Annual Meeting of EAVP, Torino, Italy.

Luo Z., Gingerich P. D., 1999. Terrestrial Mesonychia to aquatic Cetacea: Transformation of the basicranium and evolution of hearing in whales. University of Michigan Papers on Paleontology 31, 1-98.

Miyamoto, M. M., Goodman, M., 1986. Biomolecular
Systematics of Eutherian Mammals: Phylogenetic Patterns and Classification. Systematic Biology 35(2), 230-240. doi: 10.1093/ sysbio/35.2.230

Montgelard, C., Catzeflis, F. M., Douzery, E., 1997. Phylogenetic relationships of artiodactyls and cetaceans as deduced from the comparison of cytochrome $\mathrm{b}$ and $12 \mathrm{~s}$ rRNA mitochondrial sequences. Molecular Biology and Evolution 14, 550-559.

Nummela, S., Thewissen, J. G. M., 2008. The physics of sound in air and water. In Thewissen, J. G. M., and Nummela, S (Eds.), Sensory evolution on the threshold adaptations in secondarily aquatic vertebrates. University of California press, pp. 175-181.

O'Leary, M. A., 2010. An anatomical and phylogenetic study of the osteology of the petrosal of extant and extinct artiodactylans (Mammalia) and relatives. Bulletin of the American Museum of Natural History 335, 206 pp.

O’Leary, M. A., Gatesy J., 2008. Impact of increased character sampling on the phylogeny of Cetartiodactyla (Mammalia): combined analysis including fossils. Cladistics 23, 1-46.

O'Leary, M. A., Patel, B. A., Coleman, M. N., 2012. Endocranial petrosal anatomy of Bothriogenys (Mammalia, Artiodactyla, Anthracotheriidae) and petrosal volume and density comparisons among aquatic and terrestrial artiodactyls and outgroups. Journal of Paleontology 86, 44-50.

Orliac M. J., Antoine P.-O., A.-L. Charruault, Hervet S., Prodeo F., Duranthon F., 2013. Specialization for amphibiosis of Brachyodus onoideus (Artiodactyla, Hippopotamoidea) from the early Miocene of France Swiss Journal of Geosciences 106(2), 265-278 DOI 10.1007/s00015-013-0121-0.

Pearson, H. S., 1927. On the skulls of early Tertiary Suidae, together with an account of the otic region in some other primitive Artiodactyla. Philosophical Transactions of the Royal Society of London, 215, 389-460.

Reysenbach de Haan, F. W., 1960. Some aspects of mammalian hearing under water. Proceedings of the Royal Society of London, B, 152B, 54-62.

Wible, J. R., 1990. Late Cretaceous marsupial petrosal bones from North America and a cladistic analysis of the petrosal in therian mammals. Journal of Vertebrate Paleontology 10, 183-205.

Wile, J. R., 1993. Cranial Circulation and Relationships of the Colugo Cynocephalus (Dermoptera, Mammalia). American Museum Novitates 3072, 27pp.

Wible, J. R., 2011. On the Treeshrew Skull (Mammalia, Placentalia, Scandentia). Annals of Carnegie Museum 79(3), 149-230. 